\title{
Wissenschaftsgeschichte und das Revival der Begriffsgeschichte
}

\author{
Désirée Schauz
}

In den Geistes- und Kulturwissenschaften lässt sich seit kurzem ein begriffsgeschichtliches Revival beobachten, das inzwischen auch die Wissenschaftsgeschichte erfasst hat. Von dieser Forschungskonjunktur zeugen nicht nur neuere Publikationen, sondern auch Konferenzen wie die der Gesellschaft für Wissenschaftsgeschichte, die diesem Thema 2014 ihre Jahrestagung widmete. ${ }^{1}$ Die Vielfalt begriffsgeschichtlicher Ansätze, die Folge des steigenden Interesses wie auch der neuen Analysemöglichkeiten durch die wachsende Zahl an digitalisierten Quellenbeständen ist, haben Wissenschaftshistorikerinnen bis jetzt allerding nur bedingt für sich entdeckt. Die vorhandenen begriffsgeschichtlichen Studien behandeln vorrangig Aspekte der historischen Epistemologie, das heißt sie fragen nach dem Prozess der wissenschaftlichen Begriffsbildung beziehungsweise Aneignung von Begriffen, der Rolle von naturwissenschaftlichen Fachbegriffen oder Metaphern im Erkenntnisprozess sowie deren Bedeutung für die Autorität wissenschaftlichen Wissens im öffentlichen Diskurs. Konzeptionell greifen sie dabei überwiegend auf wissenschaftsphilosophische Traditionen zurückgegriffen, wie etwa auf Arbeiten von Georges Canguilhem und Hans Blumenbergs „Metaphorologie“. ${ }^{2}$ Andere etablierte Ansätze etwa aus der Geschichts- und Politikwissenschaft, deren Fokus auf der politisch-sozialen Sprache liegt, oder neuere kultur- und kommunikationswissenschaftliche Zugänge fanden dagegen in der Wissenschaftsgeschichte bisher kaum Anklang.

Es ist jedoch gerade die konzeptionelle Pluralität, die das derzeitige internationale Revival der Begriffsgeschichte kennzeichnet. Eine neue Generation von Begriffshistorikerinnen hat die alten Gräben zwischen Ideengeschichte, intellectual history und historischer Semantik und damit bestehende disziplinäre Barrieren zwischen Philosophie, Geschichtswissenschaft, Sozial-, Literatur-und Sprachwissenschaften überwunden (Steinmetz 2008). Diskursanalytische Zugänge werden mit begriffsgeschichtlichen 
verknüpft und die Metapherngeschichte, die mitunter im vorsprachlichen Bereich ansetzt, geht der Frage nach, wie aus Metaphern Begriffe werden (Bödecker 2002; Teichert 2008). Streng genommen ist Begriffsgeschichte als Etikett für die hier versammelten Ansätze inzwischen zu eng, doch die synergetischen Effekte dieser Diskussionen versprechen eine anregende Zusammenarbeit, von der auch wissenschaftsgeschichtliche Studien, die in diesem Feld nach wie vor eine Randerscheinung sind, profitieren können.

Wie andere geisteswissenschaftliche Disziplinen auch vollzog die Wissenschaftsgeschichte in den letzten Jahrzehnten mehrere turns hintereinander. Es ist noch gar nicht so lange her, dass der linguistic turn vom practical turn abgelöst wurde. Die Frage nach Praktiken hat seither zu einem fruchtbaren Perspektivwechsel geführt, mit dem Wissens- wie soziale Praxis der frühneuzeitlichen Gelehrtenkultur, Praktiken der wissenschaftlichen Objektivität und des Experimentierens sowie ganz allgemein Laborarbeit in das Blickfeld rückten. Der darauf folgende material turn brachte ebenfalls einen Perspektivwechsel und damit neuartige "Stoff-Geschichten“ etwa von Vitaminen und Hormonen hervor. Es stellt sich daher berechtigterweise die Frage, welchen erkenntnistheoretischen Mehrwert eine neuerliche Hinwendung zu Sprache und Begriffen für die Wissenschaftsgeschichte erbringen kann. Jedem turn folgte unweigerlich eine Schwerpunktverschiebung, bei der bisher für relevant gehaltene Aspekte in den Hintergrund rückten.

Die momentan in der Wissenschaftsforschung sehr populäre AkteurNetzwerk-Theorie (ANT) - um nur ein Beispiel zu nennen - hat für ihre Frage nach der Vernetzung von wissenschaftlichen und gesellschaftlichen Akteuren einerseits und Dingen andererseits, die Programme des practical wie des material turns gleichermaßen umgesetzt. Diskurse und Semantiken sind für die ANT dagegen nicht von Bedeutung, und das nicht nur weil es dabei um Interaktionen zwischen Menschen und Dingen geht. Die von Akteuren verwendete Sprache steht hier vielmehr im Verdacht, die hybriden Netzwerke nur verzerrt zu repräsentieren. Antonyme und diskursive Grenzziehungen etwa zwischen reiner und angewandter Wissenschaft oder zwischen Natur und Gesellschaft - gelten als strategisch motivierte Reinigungsdiskurse, die darauf abzielen, die tatsächliche, komplexe Forschungspraxis zu verschleiern (Latour 1998). Bruno Latour folgt zwar den gegenseitigen Zuschreibungen der Akteure im Sinne eines semiotischen Ansatzes, doch deren zeitspezifischen Bedeutungen oder ideologischen Dimensionen seien für die Analytikerin der ANT nicht von Interesse (Latour 1988: 10-11). Anstatt also die Funktion des Begriffsgebrauchs für die Kommunikation der Akteure zu untersuchen, strebt die ANT für die Beobachterrolle eine "Infrasprache“ an, „die strikt bedeutungslos bleibt", um so die in Sozialwissenschaften anzutreffende Vermischung von analytischer und Akteurssprache zu vermeiden (Latour 2007: 54). Während Latour so mit seiner „symmetrischen Anthropologie“ den 
bevormundenden Geist der kritischen Theorie in den Sozialwissenschaften vertreiben möchte, werfen ihm Kritikerinnen vor, zu entpolitisieren und die Rolle von Ideologie zu übersehen (Wahrig 2010: 195-200).

Ich möchte im Folgenden für eine erweiterte Anwendung begriffsgeschichtlicher Perspektiven in der Wissenschaftsgeschichte plädieren, um genau nach diesen Funktionen von Begriffen zu fragen, mit denen Gesellschaften über Ziele, Praktiken und Folgen von Forschung kommunizieren. Neben dem wachsenden Feld der historischen Epistemologie, in dem sich die Begriffsgeschichte bereits als eine wichtige Ergänzung erwiesen hat, sehe ich in der Analyse von "metascientfic statements" (Shapin 2001) eine weitere Möglichkeit, die Begriffsgeschichte für die Wissenschaftsgeschichte fruchtbar zu machen. Im Mittelpunkt stehen hierbei Begriffe, die sich auf übergeordnete Aspekte des neuzeitlichen Wissenschaftsverständnisses beziehen und deren semantische Zuschreibungen Ergebnisse eines wissenschaftlich-gesellschaftlichen Aushandlungsprozesses über die Forschung betreffenden Erfahrungen, Erwartungen, Ziele und Ängste sind. Dazu gehören Basiskategorien wie Wissenschaft, Wissenschaftlerinnen, Naturwissenschaften, Technik, Forschung oder Innovation, des weiteren spezifische forschungspolitische Vokabel wie reine/angewandte Wissenschaft, Grundlagen- und angewandte Forschung oder auch solche, die wissenschaftskritische Positionen ausdrücken - etwa Elfenbeinturm (Shapin 2012) oder Szientismus (Schöttler 2012). In diesen Begriffen kondensieren sich epistemische Normen und Ideale der Wissenschaft ebenso wie konkrete organisatorische und finanzielle Aspekte der Forschung sowie politische Ideologien und gesellschaftliche Werte.

Für eine Begriffsgeschichte, die auf die wissenschaftspolitische Sprache fokussiert, sind jenseits der Blumenberg'schen Metaphorologie gerade auch Ansätze der historischen Semantik in der Tradition von Reinhart Koselleck gewinnbringend, da sie für den gesellschaftlich-politischen Diskurs Deutungsangebote zum semantischen Wandel und zum Prozess der Ideologisierung machen. Die historische Semantik liefert eine geschichtstheoretische Fundierung, mit der sich erstens - basierend auf der Annahme, dass sich verschiedene Zeitschichten in Begriffe einschreiben können - neben dem semantischen Wandel auch die Persistenz wissenschaftlicher Grundvorstellungen nachvollziehen lässt. Zweitens verweist die historische Semantik auf die Verschränkung von Vergangenheitskonstruktionen und Zukunftsvisionen. Dieses temporale Bewusstsein ist für die Analyse des neuzeitlichen Wissenschaftsverständnisses von zentraler Bedeutung, da mit ihm unweigerlich Erwartungen - sei es im Sinne eines Fortschrittsglaubens oder einer Fortschrittskritik - verbunden sind. Drittens wird in der Differenz zwischen Erwartungshorizont und Erfahrungsraum ein zentraler Faktor für das Entstehen und die Bedeutung von Ideologien ausgemacht. ${ }^{3}$ Für die historische Semantik bietet die Analyse von metawissenschaftlichen Begriffen gleichfalls eine wichtige Ergänzung. Obwohl Koselleck selbst Wissenschaft und Technik 
als zentrale Faktoren des neuzeitlichen Fortschrittsdenkens benannte, fehlten die entsprechenden Begriffe im Monumentalwerk Geschichtliche Grundbegriffe. Daran haben auch Kosellecks Nachlassverwalter wie etwa Lucien Hölscher oder Willibald Steinmetz, die seine Ansätze theoretisch weiterentwickelten und um neue Themenfelder ergänzten, nichts geändert.

Im Anschluss an Koselleck lässt sich des Weiteren argumentieren, dass eine Begriffsgeschichte des neuzeitlichen Wissenschaftsverständnisses gar nicht davon ausgeht, dass Sprache Wirklichkeit im Verhältnis von eins zu eins abbildet. Begriffe sind kein Epiphänomen einer wie auch immer gearteten Wirklichkeit. Sie sind vielmehr konstitutiv dafür, wie Menschen die vorgefundene Realität sowie die diesbezüglichen Erfahrungen und Erwartungen fassen. Die kommunikative Bedeutung von Leitbegriffen liegt gerade in deren semantischen Überschuss, mit dem - unter anderem - Erfahrungsdifferenzen, widersprüchliche Erwartungen oder konfligierende Interessen überbrückt werden können. Kurzum: Die Begriffsgeschichte interessiert sich für die Polysemie von Sprache. Sprache kann Grenzen des Sag- und Denkbaren definieren, aber eben auch Kommunikations- und damit auch Handlungsspielräume eröffnen. Es kann deshalb nicht darum gehen, eine begriffsgeschichtliche Perspektive gegen eine praxeologische auszuspielen. Sprache und diskursive Praktiken sind Teil der Wissenschaft ebenso wie nichtsprachliche Praktiken und Materialität. Wichtig ist, die Verhältnisse dieser unterschiedlichen Elemente und Zugänge zueinander zu diskutieren. Semantischer Wandel oder Neologismen können beispielsweise auf Umbrüche hinweisen, bei denen etablierte Praktiken, dominante Sichtweisen sowie der eingeübte Umgang mit Materialität in Frage gestellt werden. Mehr noch: Begriffe und diskursive Praktiken spielen eine wichtige Rolle, wenn Akteure die Konstellationen und Netzwerke neu aushandeln.

Die Begriffsgeschichte von metawissenschaftlichen Konzepten ermöglicht verschiedene Forschungsperspektiven, die sowohl die disziplinäre Selbstreflexion der Wissenschaftsgeschichte erhöhen, als auch zur Reflexion über aktuelle theoretische wie forschungspolitische Debatten anregen, und zwar indem das heutige Wissenschaftsverständnis ebenso wie dessen Konzeptualisierungen durch die Wissenschaftsforschung historisiert werden. Entlang der großen theoretischen Debatten der letzten Jahre lassen sich gleich mehrere Fragekomplexe für Studien zur wissenschaftspolitischen Sprache ableiten. Das Verhältnis von Wissenschaft und Gesellschaft ist ein erster solcher Komplex, für den verschiedene Konzeptualisierungen wie beispielsweise die „co-production of science“ (Jasanoff 2004), die ANT oder in Anlehnung daran die Vorstellung von „Ressourcen für einander“ (Ash 2002) zu nennen sind. Vor allem die Interdependenz von Wissenschaft und Öffentlichkeit bzw. Politik war in den letzten Jahren Gegenstand von Studien, die sich mit der politischen Bedeutung frühneuzeitlicher Naturforschung, mit der Rolle von Wissenschaft im Zuge der kolonialen Eroberungen, Forschung im Kontext der Weltkriege 
und während des Kalten Krieges beschäftigten. ${ }^{4}$ Mal wird die Interdependenz als alltägliche Praxis aufgearbeitet, die lange Zeit vom Zerrbild der reinen Wissenschaft verdeckt blieb; mal erscheint sie als Gefahr für die wissenschaftliche Integrität.

Das Verhältnis von Wissenschaft und Gesellschaft konstituiert sich nicht zuletzt in einem permanenten Aushandlungsprozess, in dem unterschiedliche Interessen, epistemische wie gesellschaftliche Ziele und Normen, institutionelle und finanzielle Arrangements sowie die damit verbundenen Erwartungen und Erfahrungen abgeglichen werden. Sprache und Begriffe sind essentiell für diesen Aushandlungsprozess. Zu fragen ist, welche spezifischen diskursiven Funktionen Begriffe hier übernehmen. Beiträge der historischen Semantik, die sich auf Schlüsselbegriffe dieser wissenschaftspolitischen Kommunikation spezialisieren, können zeigen, wie historische Akteure zu unterschiedlichen Zeitpunkten Chancen, Herausforderungen und Probleme für Wissenschaft und Gesellschaft wahrnahmen. Sie können der Frage nachgehen, welche Differenzen und Dilemma möglicherweise überbrückt werden mussten und welche kommunikative Rolle dabei Begriffe spielten.

Ein Beispiel hierfür ist die forschungspolitische Vokabel „Grundlagenforschung". Ihre Bedeutung verdankt sie dem metaphorischen Gebrauch von "Grundlage“, mit dem sowohl das Versprechen verbunden ist, die Grundlagen einzelner Disziplinen zu erweitern, als auch das Versprechen, Grundlagen für technischen und gesellschaftlichen Fortschritt zu schaffen. Die Polysemie ermöglichte es - zumindest zeitweise -, konfligierende Interessen zu überbrücken und Freiraum für Forschung zu schaffen, deren Ergebnisse und gesellschaftliche Nützlichkeit nur schwer zu prognostizieren sind (Schauz 2014). Im Sinne des Aushandlungsprozesses lassen sich neben forschungspolitischen Begriffen aber genauso Begriffsgeschichten spezifischer epistemischer Normen wie die der Objektivität im Hinblick auf ihre gesellschaftliche Relevanz fortschreiben. In der Neuzeit entwickelte sich Wissenschaft zu einer gesellschaftlichen Wahrheitsinstanz beziehungsweise Instanz objektiver Expertise und Evidenz. Zu fragen wäre hier etwa nach dem ständigen Changieren von Wahrheits- und Objektivitätsbegriff, nach gesellschaftlichen Bedeutungszuschreibungen wissenschaftlicher Objektivität, nach möglichen politischen oder ideologischen Überformungen und danach, wie die gesellschaftlichen Erwartungen an die wissenschaftliche Expertise zurück auf den innerwissenschaftlichen Diskurs wirken.

Ein zweiter möglicher, daran anschließender Themenkomplex ist der Wandel des wissenschaftlichen Selbstverständnisses. Begriffe wie etwa „reine Wissenschaft" spielten unter anderem eine zentrale Rolle für das professionelle „identity work“ von Forschenden (Kaldewey 2013: 408-409), bei dem es erstens um innerwissenschaftliche Abgrenzungen und akademische Hierarchien ging. Zweitens bildet sich die professionelle Identität von Wissenschaftlerinnen immer auch in Auseinandersetzung mit den 
gesellschaftlichen Erwartung und Bedeutungszuschreibungen gegenüber der Wissenschaft oder einzelnen Forschungsgebieten heraus. Mit dem Konzept der reinen Wissenschaft behaupteten im 19. Jahrhundert die zunehmend akademisierten Forscherinnen ihre Unabhängigkeit von anderen gesellschaftlichen Autoritäten und Wahrheitsinstanzen (Gieryn 1999: 37-64).

Ein weiterer Aspekt des wissenschaftlichen Selbstverständnisses sind die regelmäßig wiederkehrenden Debatten über die Einheit der Wissenschaft oder das Auseinanderdriften der „zwei Kulturen“ (Snow 1959). Obwohl zuletzt der Krieg der Wissenschaften - Stichwort "Sokal hoax" - in Europa nicht so stark tobte wie auf der anderen Seite des Atlantiks, gab und gibt es auch hier Debatten über die Krise der Geisteswissenschaften. Dabei geht es inzwischen nicht mehr nur um eine Kritik an postmodernen Ansätzen oder die divergierenden Wissenskulturen der "harten" Natur- und der der "weichen" hermeneutischen Geisteswissenschaften. Angesichts des forschungspolitischen Imperativs technologischer Innovationen und ökonomischer Verwertbarkeit müssen die Geisteswissenschaften ihre gesellschaftliche Relevanz neu definieren. Derzeit fürchten vor allem britische Kolleginnen um die Konkurrenz mit den Ingenieur- und Naturwissenschaften und davor, dass die Geisteswissenschaften vor dem Ausverkauf stehen. ${ }^{5}$ Historisch gesehen, gestaltete sich das Verhältnis von Naturwissenschaften und Geisteswissenschaften sehr wechselvoll, wobei die Identitätsarbeit der materiell-empirisch orientierten Naturwissenschaftlerinnen im Zuge der Akademisierung im 19. Jahrhundert und nach dem Zweiten Weltkrieg durchaus die Nähe zur Geisteskultur suchte. Wissenschaft als kulturellen Wert zu definieren, bot gerade in Zeiten gesellschaftlicher Kritik an den Gefahren des wissenschaftlichtechnischen Fortschritts eine durchaus willkommene Entlastungsstrategie.

Für die Wissenschaftsgeschichte als Disziplin bietet die Aufarbeitung dieser Beziehungsgeschichte gleich in zweierlei Hinsicht eine Chance: Erstens wäre es ein Versuch, über die de facto häufig immer noch anzutreffende Trennung der Geschichte der Naturwissenschaften von der der Geistes- und Sozialwissenschaften zu reflektieren. Zweitens kann die Wissenschaftsgeschichte - als geisteswissenschaftliche Disziplin, die über Wissenschaft forscht - über eine Historisierung der „zwei Kulturen“ einen konstruktiven Beitrag zur Kontroverse leisten, ohne sich dabei dem Vorwurf aussetzen zu müssen, sich durch einen kritischen Fingerzeig von den Naturwissenschaften zu entfremden, wie es Bruno Latour (2004) und Lorraine Daston (2009) monierten.

Im Zeichen der von Teilen der Wissenschaftsforschung ausgerufenen technoscience Ära ist das Verhältnis zwischen Naturwissenschaft und Technik ein dritter relevanter Themenbereich, dem begriffsgeschichtliche Studien eine neue, reflexive Dimension verleihen können. Diejenigen in der Wissenschaftsgeschichte, die diesem Ruf bislang folgten, versuchten entweder den historischen Nahtstellen von Wissenschaft und Technik wie dem Instrumentenbau nachzugehen oder nach frühneuzeitlichen Ahnherren der 
technoscience zu suchen, die den Konnex zwischen Naturforschung und Technik betonten und damit das technisch-materielle Fortschrittsversprechen begründeten. Aus begriffsgeschichtlicher Sicht lassen sich andere Fragestellungen ableiten: Wie verhandelten historische Akteure das Verhältnis von Naturwissenschaften und Technik? Wann und warum kommt es zu Grenzziehungen? Welche mehr oder weniger bewussten diskursiven Strategien waren mit konzeptionellen Abgrenzungen verbunden? Sind Grenzziehungsdiskurse wirklich nur professionellem „boundary work“ (Thomas F. Gieryn) geschuldet?

Nicht zuletzt der schillernde Begriff der „technoscience“ selbst könnte aufgrund seiner Unschärfe, den unterschiedlichen Verwendungskontexten und seiner sprachpolitischen Bedeutung in theoretischen Debatten der Wissenschaftsforschung eine Historisierung erfahren. Bislang weiß man über seine begriffsgeschichtliche Karriere kaum etwas. Und schließlich lässt sich auch dieser Themenkomplex letztendlich wieder aus einer übergeordneten disziplinären Perspektive diskutieren: In der Geschichte der Abgrenzungsstrategien zwischen Naturwissenschaft und Technikwissenschaften spiegelt sich auch das Verhältnis von Technik- und Wissenschaftsgeschichte in ihren unterschiedlichen Wertzuschreibungen wider. Auch dieses Verhältnis unterliegt einem Wandel, zumindest scheint im Zeichen von technoscience - so Paul Forman (2010) - die Technikgeschichte aus dem Schatten der Wissenschaftsgeschichte hervorzutreten.

Neben einer konsequenten Historisierung aktueller theoretischer Debatten bietet die Geschichte metawissenschaftlicher Leitbegriffe außerdem die Möglichkeit der methodischen Selbstreflexion, und zwar ganz im Sinne einer historischen Quellenkritik. Begriffe wie der der Grundlagenforschung entwickelten sich aus forschungspolitischen, öffentlichen Debatten heraus und fanden dann Eingang in die analytische Perspektive der Wissenschaftsforschung. Gemäß des kulturwissenschaftlichen Ansatzes der travelling concepts (Bal 2012) sind dies Begriffe, die sich permanent zwischen öffentlichem und fachwissenschaftlichem Diskurs sowie zwischen verschiedenen Kulturen hinund her bewegen. Insofern ist Latours Sprachkritik zuzustimmen. Doch ob man dieser Verflechtung durch eine "Infrasprache“ entkommt, erscheint mir eher fraglich zu sein. Die begriffsgeschichtliche Auseinandersetzung mit dieser Verflechtung halte ich dagegen für einen gangbareren Weg.

Auch Historikerinnen benutzen in der Regel solche Begriffe mit großer Selbstverständlichkeit, ohne deren jeweiligen historischen Entstehungskontext, die zeitspezifische Verwendung und mögliche semantische Varianz zu hinterfragen. Um beim Beispiel der Grundlagenforschung zu bleiben: Für die historische Aufarbeitung der Wissenschaft während des Nationalsozialismus stellt die Unterscheidung zwischen Grundlagen- und angewandter Forschung eine analytische Kategorie dar. Und obwohl sich die Unterscheidbarkeit als schwierig erweist und mit vermittelnden Kategorien wie 
anwendungsorientierter Grundlagenforschung gearbeitet wird, versäumten es NS-Expertinnen bislang, der zeitgenössischen Verwendung entsprechender Begriffe nachzugehen und damit die Angemessenheit der eigenen Kategorien zu diskutieren. Begriffsgeschichtliche Analysen sind daher ein methodisches Instrument, um sich die semantische Varianz der analytischen Sprache der Wissenschaftsgeschichte und der historisch vorgefundenen Kategorien bewusst $\mathrm{zu}$ machen. Bislang waren metawissenschaftliche Kategorien wie Technik oder Wissenschaft meistens nur dann Gegenstand der Diskussion, wenn es um wiederkehrende disziplinäre Standortdebatten ging. Diese erschöpften sich allerdings in der Regel in etymologischen Wortdefinitionen, ohne der praktischen Verwendung der Begriffe sowohl im historischen Kontext als auch in der aktuellen Wissenschaftsforschung nachzugehen.

Neben der methodischen und theoretischen Reflexion innerhalb der Wissenschaftsgeschichte können begriffsgeschichtliche Studien schließlich auch einen Beitrag zu aktuellen forschungspolitischen Debatten leisten. Mit den Reformen der Hochschul- und Forschungslandschaft der letzten Jahre lässt sich geradezu eine Flut von begrifflichen Innovationen beobachten: Im Zeichen des Wettbewerbs werden Universitäten an ihren „Leuchttürmen“, ihrer „Exzellenz“ oder ihrem „social impact“ gemessen; Hochschulen und Disziplinen versuchen sich als "brands“ neu zu erfinden und nennen sich unternehmerische Universität, „angewandte Geschichte“ oder „public history“; die Wissenschaftsforschung erfand neue Kategorien wie „Mode 2“ oder „triple helix“, um den von ihr konstatierten Forschungswandel zu benennen; im Zug der Forderung nach mehr Demokratie und Bürgerbeteiligung in der Forschung kamen Begriffe wie „citizen science“ und „responsible innovation“ auf; und, um ein letztes Beispiel zu nennen, forschungspolitisch werden nach neuen integrativen Idealen gesucht - seien es die "grand challenges“ oder das Konzept der „frontier research“ des European Research Council. Obwohl viele dieser Begriffe relativ jung sind, lassen sie sich in ein historisch gewachsenes Feld von Begriffen der wissenschaftspolitischen Sprache einordnen. Begriffsgeschichtliche Studien können Aufschlüsse darüber geben, wie und warum diese Neologismen aufkamen, um die aktuellen Sprachpolitiken zu verstehen. Ganz generell lassen sich aus Begriffsgeschichten zur wissenschaftspolitischen Sprache Erkenntnisse für die gesellschaftliche Kommunikation über Ziele und Herausforderung von Wissenschaft und Forschung ziehen, womit die Wissenschaftsgeschichte ihre Relevanz für aktuelle forschungspolitische Debatten unterstreichen könnte.

Das Revival begriffsgeschichtlicher Ansätze bietet also für die Wissenschaftsgeschichte neben den bereits verfolgten Fragestellungen der historischen Epistemologie auch die Möglichkeit, über Wissenschaft und Forschung sowie deren gesellschaftliche Rolle auf einer allgemeineren Ebene zu reflektieren. Dafür steht eine Vielfalt an Ansätzen und Methoden zur Verfügung, deren Bandbreite dieser Beitrag nicht annähernd abbilden konnte. Die 
begriffliche (Selbst-)Reflexion besteht vor allem in einer konsequenten Historisierung von Quellenbegriffen und theoretischen Konzepten der metawissenschaftlichen Sprache. Angesichts der laufenden Debatten über die Positionierung der Wissenschaftsgeschichte innerhalb des interdisziplinären Felds der Wissenschaftsforschung betont die Disziplin dadurch ihr Alleinstellungsmerkmal und gewinnt an Eigenständigkeit: Es lassen sich sowohl aktuelle Konzepte der Wissenschaftsphilosophie als auch die Theorieangebote der Sozialwissenschaften auf ihre historische Bedingtheit hin hinterfragen, womit eine kritisch-konstruktive Distanz und das Bewusstsein für die eigene (zeitliche) Standortgebundenheit geschaffen wird.

\section{Anmerkungen}

1 Siehe: http://www.gewige-online.de/16-news/38-29-31-mai-2014-jahrestagung-der-gwgin-heidelberg.html (zuletzt aufgerufen am 2.6.2015) sowie das aus einigen Beiträgen der Tagung hervorgegangene Themenheft „Begriffsbildung - Begriffsgeschichte: Entstehung und Entwicklung wissenschaftlicher Konzepte" der Zeitschrift Berichte zur Wissenschaftsgeschichte 38,2 (2015).

2 Vgl. exemplarisch Brandt (2004); Müller/Schmieder (2008); Eggers/Rothe (2009); Borck (2013). Dieser Forumsbeitrag ist leider nicht der Ort, um einen umfassenden Literaturbericht zu leisten, weshalb ich mich auch im Folgenden auf die Nennung von nur wenigen zentralen Titeln beschränke.

3 In den letzten Jahren sind viele neue Editionen mit zentralen Texten von Koselleck, meistens ergänzt durch Aufsätze von Weggefährten und Schülern, erschienen. Zum Einstieg würde ich jedoch immer noch folgende Textsammlung empfehlen: Koselleck (1989). Aktuelle Beiträge und Diskussionen zur Historischen Semantik finden sich vor allem in der Zeitschrift Contributions to the History of Concepts.

4 Gemeint sind hier erstens ältere Arbeiten, die bereits im Zuge der „Social Construction of Science"-Bewegung die politische Bedeutung der frühneuzeitlichen Naturforschung für Gesellschaften, die durch konfessionelle Spannungen und moderne Staatsbildung gekennzeichnet waren, untersuchten. Zweitens gibt es inzwischen eine Vielzahl von Studien, die insbesondere die Rolle wissenschaftlicher Disziplinen für den Aufbau des British Empire untersucht haben. Die deutsche Kolonialgeschichte zieht hier inzwischen nach. Schließlich für Deutschland im 20. Jahrhundert sind vor allem die Großprojekte zur Aufarbeitung der Geschichte der Kaiser-Wilhelm-Gesellschaft im Nationalsozialismus und der (Vor-)Geschichte der DFG zu nennen.

5 Siehe exemplarisch den Kommentar von Terry Eagelton: http://www.chronicle.com/ article/The-Slow-Death-of-the/228991 (zuletzt aufgerufen am 2.6.2015).

\section{Literatur}

Ash, Mitchell G., 2002. Wissenschaft und Politik als Ressourcen für einander. In: Rüdiger vom Bruch/Brigitte Kaderas, Hg., Wissenschaften und Wissenschaftspolitik. Bestandsaufnahmen zu Formationen, Brüchen und Kontinuitäten im Deutschland des 20. Jahrhunderts. Stuttgart: Franz Steiner, 31-51.

Bal, Mieke, 2012. Travelling Concepts in the Humanities. A Rough Guide. Toronto/Buffalo/ London: University of Toronto Press. 
Bödecker, Hans Erich, Hg., 2002. Begriffsgeschichte, Diskursgeschichte, Metapherngeschichte. Göttingen: Wallstein Verlag.

Borck, Cornelius, Hg., 2013. Hans Blumenberg beobachtet: Wissenschaft, Technik und Philosophie. Freiburg [u.a.]: Alber.

Brandt, Christina, 2004. Metapher und Experiment: Von der Virusforschung zum genetischen Code. Göttingen: Wallstein.

Daston, Lorraine, 2009. Science Studies and the History of Science. Critical Inquiry 35(4), $798-813$.

Eggers, Michael/Rothe, Matthias, Hg., 2009. Wissenschaftsgeschichte als Begriffsgeschichte. Terminologische Umbrüche im Entstehungsprozess der modernen Wissenschaften. Bielefeld: Transcript Verlag.

Forman, Paul, 2010. (Re)cognizing Postmodernity: Helps for Historians - of Science Especially. Berichte zur Wissenschaftsgeschichte 33, 157-175.

Gieryn, Thomas F., 1999. Cultural Boundaries of Science. Credibility on the Line. Chicago/London: University of Chicago Press.

Jasanoff, Sheila, Hg., 2004. States of Knowledge. The Co-production of Science and Social Order. London/New York: Routledge.

Kaldewey, David, 2013. Wahrheit und Nützlichkeit: Selbstbeschreibungen der Wissenschaft zwischen Autonomie und gesellschaftlicher Relevanz. Bielefeld: transcript.

Koselleck, Reinhart, 1989. Vergangene Zukunft: Zur Semantik geschichtlicher Zeiten. Frankfurt a. M.: Suhrkamp.

Latour, Bruno, 1988. The Pasteurization of France. Cambridge, MA/London: Harvard University Press.

Latour, Bruno, 1998. Wir sind nie modern gewesen: Versuch einer symmetrischen Anthropologie. Frankfurt a. M.: Fischer.

Latour, Bruno, 2004. Why Has Critique Run out of Steam? From Matters of Fact to Matters of Concern. Critical Inquiry 30(2), 225-248.

Latour, Bruno, 2007. Eine neue Soziologie für eine neue Gesellschaft: Einführung in die AkteurNetzwerk-Theorie. Frankfurt a. M.: Suhrkamp.

Müller, Ernst/Schmieder, Falko, Hg., 2008. Begriffsgeschichte der Naturwissenschaften. Zur historischen und kulturellen Dimension naturwissenschaftlicher Konzepte. Berlin/New York: Walter de Gruyter.

Schauz, Désirée, 2014. What is Basic Research? Insights from Historical Semantics. Minerva 52(3), 273-328.

Schöttler, Peter, 2012. Szientismus. Zur Geschichte eines schwierigen Begriffs. NTM 29(4), $245-269$.

Shapin, Steven, 2012. The Ivory Tower. The History of a Figure of Speech and its Cultural Uses. British Journal for the History of Science 45(1), 1-27.

Shapin, Steven, 2001. How to be Antiscientific? In: Jay A. Labinger/H.M. Collins, Hg., The One Culture? A Conversation about Science. Chicago: University of Chicago Press, 99-115.

Snow, Charles P., 1959. The Two Cultures and the Scientific Revolution. Cambridge: Cambridge University Press.

Steinmetz, Willibald, 2008. Vierzig Jahre Begriffsgeschichte - The State of the Art. In: Heidrun Kämper/Ludwig M. Eichinger, Hg., Sprache - Kognition - Kultur. Sprache zwischen mentaler Struktur und kultureller Prägung. Berlin/New York: Walter de Gruyter, 174-197.

Teichert, Dieter, 2008. Haben naturwissenschaftliche Begriffe eine Geschichte? Anmerkungen zum Zusammenhang von Metaphorologie und Begriffsgeschichte bei Hans Blumenberg. In: Ernst Müller/Falko Schmieder, Hg., Begriffsgeschichte der Naturwissenschaften. Zur historischen und kulturellen Dimension naturwissenschaftlicher Konzepte. Berlin/New York: Walter de Gruyter, 97-116.

Wahrig, Bettina, 2010. Eine Frage der Politik: Wissenschaft und Ideologie im 21. Jahrhundert. Berichte zur Wissenschaftsgeschichte 33(2), 193-210. 
Wissenschaftsgeschichte Und Das ReVIVAl der Begriffsgeschichte

Désirée Schauz

c/o Professur Technikgeschichte

Technische Universität München

c/o Deutsches Museum, Museumsinsel 1

80538 München

Germany

E-Mail: Desiree.Schauz@mytum.de 